\title{
La no revictimización en delitos sexuales en el sistema educativo nacional como garantía constitucional
}

\section{Failure to re-victimize sexual crimes in the national educational system as a constitutional guarantee}

\author{
Mireya Paulina Cabrera-Marín \\ mireya.cabrera@psg.ucacue.edu.ec \\ Universidad Católica de Cuenca, Cuenca \\ Ecuador \\ https://orcid.org/0000-0001-6353-7129 \\ Juan Carlos Erazo-Álvarez \\ jcerazo@ucacue.edu.ec \\ Universidad Católica de Cuenca, Cuenca \\ Ecuador \\ https://orcid.org/0000-0001-6480-2270 \\ Cecilia Ivonne Narváez-Zurita \\ inarvaez@ucacue.edu.ec \\ Universidad Católica de Cuenca, Cuenca \\ Ecuador \\ https://orcid.org/0000-0002-7437-9880 \\ Camilo Emanuel Pinos-Jaén \\ cpinosj@ucacue.edu.ec \\ Universidad Católica de Cuenca, Cuenca \\ Ecuador \\ https://orcid.org/0000-0002-0934-8471
}

Recibido: 12 de abril de 2020

Revisado: 26 de abril de 2020

Aprobado: 01 de mayo de 2020

Publicado: 17 de junio de 2020 


\title{
RESUMEN
}

La investigación ha demostrado que el artículo 6 del Acuerdo Ministerial No. MINEDUCMINEDUC-2017-00052-A, violenta la garantía Constitucional de no re-victimización. El objetivo es proponer la adecuación normativa del referido artículo a la Constitución de la República del Ecuador, para evitar la re victimización de las niñas niños y adolescentes víctimas de delitos sexuales detectados dentro de las Unidades Educativas. La investigación fue de tipo mixta, con énfasis en lo cualitativo. Se encontraron posiciones y criterios divididos, respecto de sí las intervenciones que realiza el Departamento de Consejería Estudiantil incumple el mandato Constitucional, contraponiéndose a lo dispuesto por la Carta Magna, no obstante, sobresale la consideración de que este departamento, no está facultado para realizar intervenciones a las víctimas de delitos sexuales. Finalmente es necesario realizar una reforma al 6 del Acuerdo Ministerial No. MINEDUC-MINEDUC-2017-00052-A, respecto de las intervenciones que realiza el DECE con los estudiantes agraviados.

Descriptores: Derecho constitucional; violencia; doctrina; sistema educativo; investigación. (Palabras tomadas del Tesauro UNESCO).

\begin{abstract}
The investigation has shown that Article 6 of Ministerial Agreement No. MINEDUCMINEDUC-2017-00052-A, violates the Constitutional guarantee of not re-victimization. The objective is to propose the normative adaptation of the referred article to the Constitution of the Republic of Ecuador, to avoid the re-victimization of girls, boys and adolescents victims of sexual crimes detected within the Educational Units. The research was mixed, with an emphasis on qualitative. Divided positions and criteria were found, regarding whether the interventions carried out by the Student Counseling Department violate the Constitutional mandate, contrary to the provisions of the Magna Carta, however, the consideration that this department is not empowered to carry out interventions stands out. to victims of sexual crimes. Finally, it is necessary to carry out a reform to 6 of the Ministerial Agreement No. MINEDUC-MINEDUC-2017-00052-A, regarding the interventions carried out by the DECE with the aggrieved students.
\end{abstract}

Descriptors: Constitutional right; Violence; ideologies; educational systems; research. (Words taken from the UNESCO Thesaurus). 


\section{INTRODUCCIÓN}

Las niñas, niños y adolescentes, tienen derecho a que su integridad física, psicológica y sexual sea respetada, protegida y reparada en el caso de su vulneración; consecuentemente no podrán ser re-victimizados en el ámbito educativo, administrativo, judicial, por citar. La aplicación de protocolos de atención a las víctimas de delitos sexuales, deben ser conocidos por, inter alia, los servidores públicos y empleados privados que trabajan en las Unidades Educativas, en el ámbito de salud, servidores judiciales.

En el caso de los delitos sexuales que tienen como víctimas a estudiantes, se debe evitar la re-victimización de la niña, niño o adolescente, puesto que es ultrajante para la víctima tener que iniciar una especie de vía crucis, que inicia con la narración de los hechos de los cuales se desprende información respecto a quien acudió por primera vez para contar lo sucedido, para posteriormente acudir ante la máxima autoridad de la Unidad Educativa, para correr traslado y comunicar al Departamento de Consejería Estudiantil (DECE) o en el caso de no contar con el DECE, la víctima deberá recibir los servicios especializados en el Ministerio de Salud.

Ahora bien, esto sucede en el ámbito educativo-administrativo, sin embargo, se inobserva lo dispuesto en el artículo 422 del Código Orgánico Integral Penal (COIP) por cuanto no siempre se deriva a la Fiscalía inmediatamente lo cual implica, que ciertas actuaciones previas al inicio de la investigación se obstaculicen, como por ejemplo la valoración médica y psicológica a la víctima.

Esto implica que la víctima realice doble declaración una receptada en el DECE y la segunda dentro de la fase pre procesal en la Fiscalía, a lo cual podríamos sumarle, que en ciertos casos el testimonio anticipado receptado en la cámara de Gessell y la intervención de Instituciones que trabajan con víctimas de delitos sexuales, configurarían el recordatorio de los hechos hasta por cuatro ocasiones.

Es también relevante indicar que la Constitución de la República del Ecuador (CRE) reconoce tres tipos de garantías constitucionales: Garantías normativas o abstractas; garantías de políticas públicas, servicios públicos y participación ciudadana; y, garantías 
jurisdiccionales. En este contexto y por ser materia de la presente investigación las garantías normativas tienen por objeto la adecuación formal y material de la norma infra constitucional a la Constitución; consecuentemente, como se pudo observar supra, no existe la adecuación a la que se refiere la Constitución en el artículo 6 del Acuerdo Ministerial No. MINEDUC-MINEDUC-2017-00052-A.

Según (Dupret \& Nathalia, 2013) la re-victimización es el resultado de la implementación de procedimientos de atención inadecuados que trazan una ruta llena de obstáculos, y que no respetan la vivencia penosa de la víctima, reactivando emociones negativas en relación con el delito y propiciando una repetición de la experiencia de violencia. (p. 102) La falta de adecuación material del artículo 6 del Acuerdo Nro. MINEDUC-MINEDUC2017-00052-A a la Constitución de la República del Ecuador, se evidencia la necesidad de que el estado reconozca y garantice a las niñas niños y adolescentes el derecho a la integridad personal, que incluye la integridad física, psíquica, moral y sexual, disponiendo así que las víctimas de infracciones penales deben gozar de protección especial, garantizándoles de esta forma su no re victimización (Asamblea Constituyente, 2008).

En relación a la problemática expuesta, el objetivo del presente artículo es proponer la adecuación normativa mediante reforma del artículo 6 del Acuerdo Nro. MINEDUCMINEDUC-2017-00052-A a la Constitución de la República del Ecuador que evite la re victimización de las niñas niños y adolescentes víctimas de delitos sexuales detectados dentro de las Unidades Educativas en el Ecuador.

\section{Referencial teórico}

\section{La Garantía Constitucional de no re-victimización en el Ecuador}

La doctrina jurídica al referirse a los derechos fundamentales describe cualidades, valores esenciales, de permanencia en el ser humano los cuales deben ser protegidos en el ámbito jurídico y son reconocidos por el ordenamiento jurídico positivo a partir de la Constitución, consecuentemente las garantías constitucionales resultan ser los medios o instrumentos jurídicos de los que la Constitución dota a las sociedades a efecto de que estas sostengan y defiendan sus derechos frente a posibles vulneraciones (Chiriboga \& 
Salgado , 1995). Como resultado de ello se colige que la Constitución en el Ecuador es garantista de derechos y es el estado el obligado a cumplir y hacer cumplir los mismos. Siguiendo el mismo orden de ideas, la Constitución de la República del Ecuador, en el artículo 78 establece que a las víctimas de infracciones penales se les debe garantizar su no re victimización, particularmente en la obtención y valoración de pruebas, adoptando incluso mecanismos de reparación integral para llegar al conocimiento y verdad de los hechos (Asamblea Constituyente, 2008). En este mismo contexto la Convención de los Derechos de Niñas, Niños y Adolescentes, describe claramente las medidas y formas de protección a los niños víctimas de delitos, para lo cual los estados que son parte de la Convención han adquirido compromisos y adoptado medidas (Organización de las Naciones Unidas, 1989).

Según García , Guedes , \& Knerr (2013) la investigación de delitos sexuales cometidos en contra de niñas, niños y adolescentes resulta compleja puesto que aun constituye una especie de tabú y difícil de revelar en muchos entornos, no siendo menos cierto que el abuso sexual en la niñez sucede en todos los países en los que se ha estudiado detenidamente sobre ello.

De la revisión de la legislación vigente en el Ecuador, en el Código Orgánico Integral Penal, no se establece un concepto de víctima, siendo considerada un sujeto procesal al igual que la persona procesada, su defensa y la fiscalía, no obstante según la doctrina en Derecho Penal la víctima es uno de los elementos del delito, ya que se constituye en la persona natural o jurídica sobre quien se comete la infracción o se ejecuta una acción destructora, resultando ser quien padece el daño (Marquez-Cárdenas, 2011).

Ahora bien, al hablar de víctimas de delitos sexuales, sean niñas niños o adolescentes, se debe hacer un paréntesis para analizar sus derechos constitucionales vulnerados, ya que no solo nos encontramos frente a víctimas que resultan agraviadas por el cometimiento de un delito, sino que son víctimas del que a mi discernimiento personal es uno de los delitos más infames, que afecta a la integridad, al desarrollo y a la humanidad de la persona, se observa también que la CRE (2008) dentro del numeral 3 del artículo 66 reconoce y garantiza el derecho a la integridad personal, que incluye la integridad 
física, psíquica, moral y sexual, vinculado a garantizar de igual forma el derecho a una vida libre de violencia en el ámbito público y privado.

Volviendo la mirada hacia el derecho a la integridad, no es menos cierto que se encuentra ligado desde sus orígenes al estado constitucional, identificándolo con el derecho a la dignidad o derecho al trato digno, generando así posiciones protegidas por el derecho a la integridad, considerándolo un verdadero derecho fundamental, dado que aun cuando no existía doctrina al respecto se ha discutido sobre la existencia del mismo (CanosaUsera, 2006).

La Convención de los Derechos del Niño (1989), trae consigo a la doctrina de protección integral, al indicar que los estados parte están en la obligación de adoptar todas las medidas necesarias, sean estas de tipo legislativas, administrativas, sociales y educativas, que sean apropiadas para evitar cualquier tipo de menoscabo en la integridad del niño. Haciendo eco de ello el Código de la Niñez y Adolescencia [CNA] (2003), consagra como derechos de las niñas niños y adolescentes el respeto de su integridad personal, física, psicológica, cultural, afectiva y sexual (Romero-Heras, Erazo-Álvarez, Pinos-Jaén, \& Narváez-Zurita, 2020).

Otra tarea prioritaria es adentrarnos en el principio de interés superior del niño, el cual es considerado como el eje fundamental de cada proceso en el que se cuenta con la intervención de un niño, una niña o un adolescente, constituyéndose como principio integrante del sistema de protección de los derechos de este grupo vulnerable (LópezContreras, 2015). Se observa también que dentro de la Carta Magna se exige el respeto del interés superior de las niñas, niños y adolescentes, indicando que sus derechos prevalecerán sobre los de otras personas.

Retomando la idea inicial, cualquier víctima de un delito tiene derecho a que se le precautele su integridad respetando la garantía de no re-victimización y más aún si hablamos de niñas niños y adolescentes víctimas de delitos sexuales, para lo cual se debe tener presente que todo el aparataje estatal en sus diferentes ámbitos y competencias debe propender a conocer y manejar protocolos de actuación inmediata ante estos hechos, los cuales deben asegurarle a la víctima que no tendrá que revivir los 
mismos bajo ninguna circunstancia (Torres-Pérez, Narváez-Zurita, Pinos-Jaén, \& ErazoÁlvarez, 2020).

\section{Análisis Normativo del Art. 6 del Acuerdo No. Mineduc-Mineduc-2017-00052-A}

El Ministerio de Educación es la cartera de estado que regenta el sistema educativo en el país, el cual en cumplimiento de los mandatos constitucionales debe garantizar las acciones pertinentes a fin de prevenir, erradicar e incluso sancionar las infracciones de tipo sexual, con esta finalidad y de la revisión de los considerandos se advierte la expedición del Acuerdo No. Mineduc-Mineduc-2017-00052-A, que corresponde al Instructivo de actuación, para la atención a niños, niñas y adolescentes víctimas de violencia sexual cometidas o detectadas en los establecimientos del sistema educativo nacional y los procesos para la investigación y sanción.

El artículo 154 de la CRE, determina que entre las atribuciones de las ministras y ministros de Estado, les corresponde ejercer la rectoría de las políticas públicas del área a su cargo y expedir los acuerdos y resoluciones administrativas que requiera su gestión (Asamblea Constituyente, 2008). Con el objeto de analizar de manera jurídica el referido acuerdo ministerial, cabe precisar que en el Ecuador existe la supremacía de la Constitución de la República (2008) y con ello la obligatoriedad de que las normas y actos del poder público mantengan conformidad con las disposiciones constitucionales, de lo contrario estos carecen de eficacia jurídica, para tal efecto es necesario conocer lo contenido en el artículo 6 del Acuerdo No. Mineduc-Mineduc-2017-00052-A (FalconíBenítez, 2017), el cual prevé:

Identificada la infracción de tipo sexual la máxima autoridad dispondrá al Departamento de Consejería Estudiantil (DECE), preste el apoyo psicológico y emocional al estudiante agraviado, converse con la familia y miembros de la comunidad académica a fin de brindar toda la protección que se requiera.

Tanto las primeras intervenciones de atención, así como las siguientes, por parte del personal especializado se registrarán por escrito y se mantendrán en un expediente personalizado y confidencial en custodia del director 0 responsable del DECE. En caso que la Instituciones Educativas no tengan un DECE, la autoridad del establecimiento se apoyará en el docente tutor y en los servicios especializados de asistencia psicológica del Ministerio de Salud. 
Mireya Paulina Cabrera-Marín; Juan Carlos Erazo-Álvarez; Cecilia Ivonne Narváez-Zurita; Camilo Emanuel Pinos-Jaén

Ahora bien, conocemos que la CRE está concebida en un aspecto más material que formal y esto obedece a que existe un amplio desarrollo en cuanto a los derechos fundamentales, respecto de su reconocimiento y protección, ampliando así la visión de su supremacía (Rodríguez M. F., 2011) por lo tanto, todas las normas jurídicas deben contener parámetros establecidos constitucionalmente. Dentro de esta perspectiva, no es menos cierto que el Art. 6 del Acuerdo No. Mineduc-Mineduc-2017-00052-A, debe guardar absoluta armonía con lo contenido en la CRE, de manera específica con la garantía de no re-victimización y el derecho a la integridad con el que se encuentra ligado.

\section{Análisis de casos que demuestran la vulneración de la garantía de no re- victimización.}

Para abordar este punto, a efectos de dar una visión real, se precisa analizar casos ocurridos dentro del Cantón Cuenca de la Provincia del Azuay, los cuales se encuentran en etapa procesal de llamamiento a juicio y sentencia condenatoria, casos que se enmarcan dentro de lo que a través del presente artículo de investigación se pretende demostrar.

\section{Caso 1}

La adolescente NN de 17 años de edad, quien además posee discapacidad intelectual, acude regularmente a una entidad educativa especializada, lugar en el cual es víctima de abuso sexual (tocamiento de partes íntimas) hecho perpetrado por un docente de la institución, cuando la madre de la víctima logra detectar lo sucedido con su hija, en primera instancia pone en conocimiento de la Directora, quien dispone inmediatamente que actúe el DECE, como intervención por parte de quienes abordaron a la estudiante agraviada proceden a pedirle que indique quien fue? cómo fue? quién le tocó? en donde le tocó? incluso requiriendo a la adolescente quien además es una persona que posee discapacidad intelectual, les lleve al lugar de los hechos haciendo una especie de reconstrucción de los hechos, días después ante la solicitud de una respuesta por parte de la progenitora de NN, la Directora de la unidad educativa, pone la respectiva denuncia en la Fiscalía General del Estado, a fin de que proceda con la investigación que le 
corresponde de conformidad a lo dispuesto en el Art. 195 de la CRE.

\section{Caso 2}

De igual manera, expondremos lo ocurrido con la niña NN de 8 años de edad, quien fue ultrajada sexualmente por su tío, debido a que su agresor era un familiar, la menor decide comentar sobre el hecho a su maestra de clase, quien apegada a lo dispuesto en el acuerdo ministerial, materia del presente artículo, lleva a la niña donde la psicóloga del DECE, ante quien NN vuelve a narrar los actos perpetrados por su tío en contra de su integridad física y sexual, posterior a ello se pone en conocimiento de la Autoridad educativa y se deriva la denuncia a Fiscalía General del Estado, en donde es prescindible realizar a la niña la valoración médica y psicológica, en la que necesariamente la niña debe volver a comentar sobre lo ocurrido, para posterior a ello rendir su testimonio anticipado, relatando por cuatro ocasiones ante diversas personas y en escenarios diferentes todo lo sucedido (Zhindón-Idrovo, Erazo-Álvarez, Pozo-Cabrera, \& NarváezZurita, 2020).

En este sentido, al efectuar acciones probatorias con las que se tiende a reconstruir la conducta reprochable, ineludiblemente se genera en la víctima, un recordatorio del daño sufrido, una vuelta al pasado, con ello, una re victimización practicada por los instrumentos sociales que teóricamente están llamados a reparar el daño sufrido (Moscoso-Parra, Correa-Calderón , \& Orellana-Izurieta , 2018).

La Corte Interamericana de Derechos Humanos (CIDH) dentro del Caso V.R.P., V.P.C. y otros vs. Nicaragua (2018), centró su análisis sobre la cuestión de si las investigaciones y el proceso penal iniciados a nivel interno por el estado, debido a la denuncia por violación sexual interpuesta por la madre de V.R.P., cumplieron con el deber de debida diligencia y de no re victimización en investigaciones y proceso penales por violencia sexual en perjuicio de una niña. De igual manera la Corte Interamericana estimó positivo y favorable para las víctimas de delitos sexuales la utilización de dispositivos exclusivos como la cámara de Gesell o circuitos cerrados de televisión, esto con el propósito de minimizar cualquier efecto revictimizante, concluyendo de esta forma la CIDH que el Estado de Nicaragua es responsable entre otras situaciones de la revictimización de la 
niña V.R.P., quien fué violentada sexualmente por su padre.

Finalmente los casos y aspectos analizados, permiten concluir que para un niño, niña o adolescente víctima de un delito sexual, resulta completamente perjudicial para su integridad física y emocional, revivir los hechos a través de la narración de los mismos, a más de una persona y en más de un espacio físico, violentando así el estado, a través de sus servidores la consagrada garantía constitucional de no re-victimización.

\section{MÉTODO}

En la presente investigación se ha optado por un tipo de diseño no experimental descriptivo, debido a que no se ha manipulado las variables y se han observado fenómenos, tal como estos suceden en su contexto natural, observando situaciones ya existentes, las cuales no se han sido provocadas intencionalmente dentro de esta investigación (Hernández-Sampieri, Collado, \& Baptista, 2014).

De esta manera para la recolección de información, se utilizaron herramientas cualitativas de revisión bibliográfica, sobre el tema de la investigación, a través de una macro búsqueda que faculta la identificación de los documentos referentes al tema de investigación, así como la categorización y organización con el objeto de analizar las principales características (Gómez-Luna, Fernando-Navas, Aponte-Mayor, \& BetancourtBuitrago, 2014).

El método aplicado es inductivo-deductivo porque su base es la repetición de los hechos y fenómenos de la realidad, para encontrar los rasgos comunes de un grupo definido, llegando así a conclusiones de los aspectos de lo caracterizan, puesto que su generalización posee una base empírica (Rodríguez A. , 2017).

De este modo el enfoque empleado es mixto, ya que permite entender y dimensionar el hecho de manera más profunda, extensa y compleja, permitiendo así observar la realidad con mejores herramientas de lectura. Cabe considerar por otra parte que resultó de absoluta relevancia la combinación de estos métodos debido a que ha permitido desde el campo del derecho y la jurisprudencia realizar aportes en cuanto a debates profesionales y entendidos en leyes (Villabella, 2015). 


\section{Universo de estudio y tratamiento muestral}

Se utilizó el muestreo por conveniencia que es una técnica de muestreo no probabilístico y aleatorio que está formado por los casos disponibles a los cuales se tiene acceso y la disponibilidad de las personas de formar parte de la investigación, que en este caso particular fueron 18 funcionarios públicos, entre agentes fiscales, secretarios de fiscalías, miembros de la Junta Cantonal de Protección de Derechos, analistas jurídicos de la Coordinación No. 6 de Educación.

\section{Tratamiento estadístico de la información}

Se obtuvieron datos a mediante cuestionarios y a través de los formularios realizados por Google (https://docs.google.com/forms), estos fueron procesados en tablas de datos que recopilan las respuestas de las personas involucradas en la investigación, y se tabulan los resultados más importantes en el programa Microsoft Excel versión 2019 (ErazoÁlvarez \& Narváez-Zurita, 2020).

\section{RESULTADOS}

Los resultados generados a través del instrumento de recopilación de información se expresan a continuación:

\section{Obligatoriedad de denunciar}

Del análisis realizado al contenido del artículo 6 del Acuerdo No. Mineduc-Mineduc-201700052-A, se ha preguntado a las personas encuestadas si con la existencia del referido instrumento legal, se estaría inobservando la obligatoriedad de denunciar de manera inmediata cualquier hecho de violencia sobre el cual se llegue a tener conocimiento, el $55,56 \%$ respondió que no consideran que exista tal inobservancia, ya que por parte de las instituciones educativas, si existe la denuncia pertinente ante las autoridades competentes cuando tienen conocimiento de la comisión de delitos. Hay sin embargo el $44,44 \%$ que manifiesta que el citado artículo sí pasa por alto este mandato legal de denunciar, debido a que a criterio de algunas personas encuestadas la cartera de estado realiza omisiones que en algunos casos pueden llegar incluso a entorpecer la 
investigación y el proceso judicial a efectuarse.

Por otro lado el $100 \%$ de los funcionarios encuestados, coincidieron que es correcto y necesario que, de manera inmediata en cuanto llegan a tener conocimiento de que dentro de la institución educativa existe una víctima de delito sexual se debe denunciar ante el órgano competente que es la Fiscalía General del Estado.

\section{Intervención a víctimas}

De conformidad con el Acuerdo Ministerial, materia de la presente investigación el DECE queda facultado para realizar varias intervenciones con respecto a los estudiantes agraviados, las cuales parten desde brindar apoyo psicológico y emocional, hablar con sus familiares y comunidad educativa, otorgar protección, hasta mantener un expediente con la categorización de confidencial en custodia del director o responsable del antes indicado departamento, ante ello del universo total de los funcionarios encuestados, el $55,56 \%$ reflexionan que al realizar ese tipo de intervenciones se configura la revictimización debido a que dentro de las intervenciones se cuestiona a la o el estudiante agraviado sobre los hechos sucedidos, realizando diligencias que se repetirán durante el proceso penal, además de que a criterio de las personas encuestadas el personal del DECE no tiene la preparación para ser un organismo de primera acogida. Por el contrario el $44,44 \%$ piensa que no existe tal re-victimización ya que incluso este departamento de la entidad educativa puede servir como un filtro para direccionar si se trata de un delito o se debe derivar a otra instancia.

\section{Principio de Reserva}

Tal como mencionamos en el acápite anterior el DECE puede mantener un expediente personalizado y confidencial en donde se registrarán las intervenciones que se realicen con la víctima, al conocer que existe el principio de reserva y más aún en delitos sexuales que tienen como víctimas a grupos vulnerables, el 55,56\% de los encuestados respondió que a todas luces se vulnera tal reserva, opinión diferente tuvo el $44,44 \%$ ya que dicen que el expediente que se encuentra en poder del DECE tiene la figura de confidencial y 
Iustitia Socialis. Revista Arbitrada de Ciencias Jurídicas.

Año V. Vol. V. N9. Julio - Diciembre 2020

Hecho el depósito de Ley: FA2016000064

ISSN: 2542-3371

FUNDACIÓN KOINONIA (F.K). Santa Ana de Coro, Venezuela

Mireya Paulina Cabrera-Marín; Juan Carlos Erazo-Álvarez; Cecilia Ivonne Narváez-Zurita; Camilo Emanuel Pinos-Jaén

no constituye un expediente penal.

\section{Acompañamiento a la víctima (estudiante)}

Al respecto de que el DECE brinde acompañamiento a la víctima (estudiante), durante el proceso pre procesal e incluso procesal penal, el 44,44\% estima que es positivo, ya que el estudiante está relacionado con los funcionarios de su entidad educativa y eso genera una especie de confianza, realizando así contingencia en las víctimas. Hay sin embargo el 55,56\% que manifiesta que no tiene una connotación favorable para la víctima el hecho de que el DECE le proporcione acompañamiento.

\section{Necesidad de adecuar el artículo 6 del Acuerdo No. Mineduc-Mineduc-2017-00052- A a la CRE}

Conocido es por todos quienes habitamos en el territorio nacional la supremacía de la Constitución de la República del Ecuador (2008) y la obligatoriedad de que las normas y actos del poder público mantengan conformidad con las disposiciones constitucionales, de lo contrario carecen de eficacia jurídica. A este respecto el $55,56 \%$ de las personas encuestadas suponen que el artículo 6 del Acuerdo No. Mineduc-Mineduc-2017-00052A, debe ser adecuado a la norma Constitucional, al inverso del $44,44 \%$. 
Iustitia Socialis. Revista Arbitrada de Ciencias Jurídicas.

Año V. Vol. V. N9. Julio - Diciembre 2020

Hecho el depósito de Ley: FA2016000064

ISSN: 2542-3371

FUNDACIÓN KOINONIA (F.K). Santa Ana de Coro, Venezuela

Mireya Paulina Cabrera-Marín; Juan Carlos Erazo-Álvarez; Cecilia Ivonne Narváez-Zurita; Camilo Emanuel Pinos-Jaén

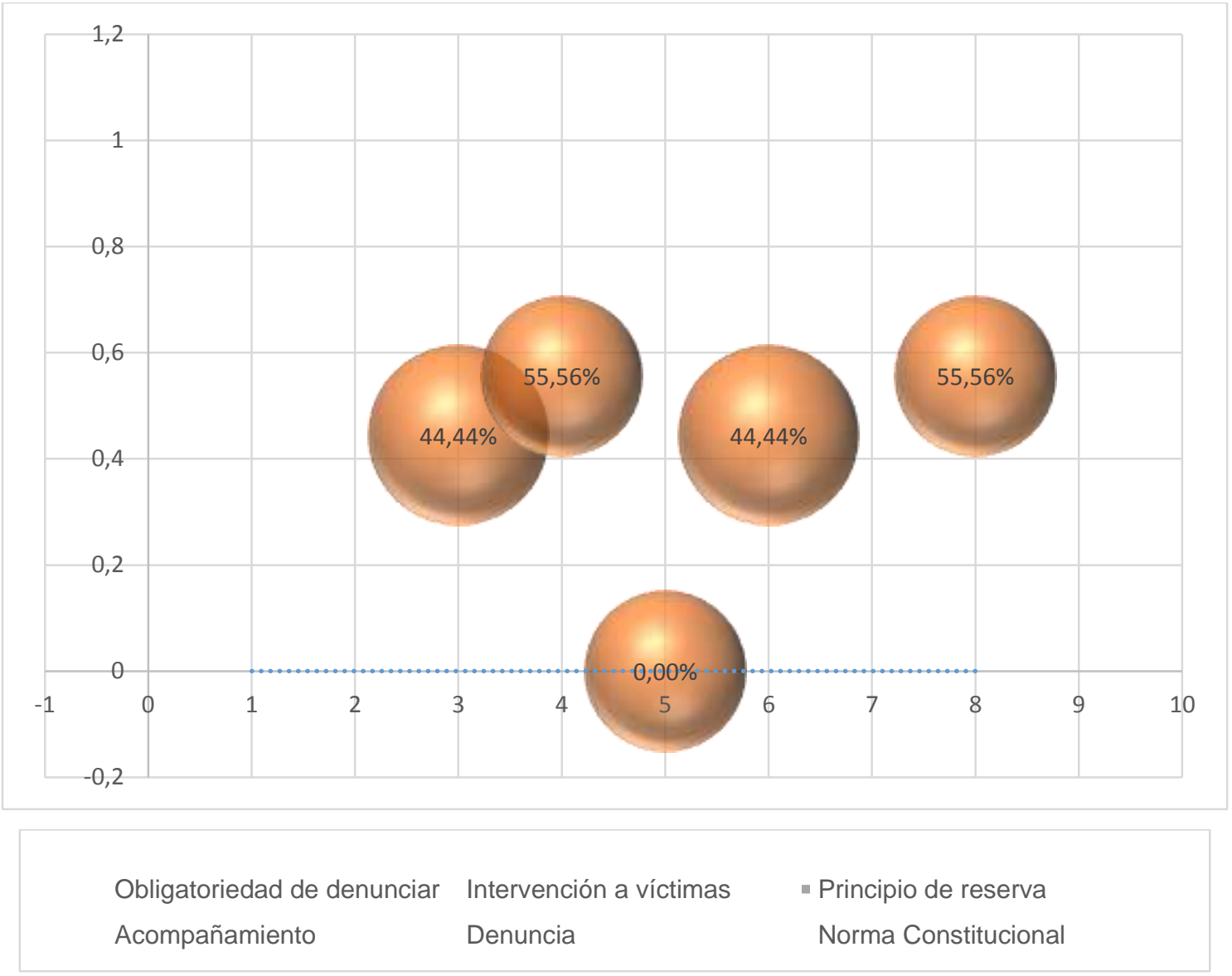

Figura 1. Resultados

\section{PROPUESTA}

Reforma del artículo No. 6 del Acuerdo Ministerial No. MINEDUC-MINEDUC-201700052-A

\section{Objeto}

El presente artículo de investigación tiene por objeto evidenciar la existencia de vulneración de la garantía constitucional de no re-victimización, lo cual se halla configurado en las diferentes intervenciones que realiza el DECE con los estudiantes que son víctimas de delitos sexuales. 


\section{Antecedentes}

De la revisión del artículo 6 del Acuerdo Ministerial No. MINEDUC-MINEDUC-201700052-A, se advierte la falta de adecuación del referido artículo del Acuerdo Ministerial a la Constitución de la República del Ecuador, por lo que se trabajó en la elaboración del presente artículo de investigación, el cual trae consigo una propuesta de reforma.

\section{Exposición de motivos}

En la vida diaria de quienes nos encontramos de alguna forma vinculados al conocimiento de casos de violencia sexual, que tienen como víctimas a niñas, niños o adolescentes, cuyo cometimiento o detección se realizó dentro de una institución del sistema educativo del país, sabemos que los agraviados son sometidos a una constante re victimización, durante el proceso administrativo y judicial.

La CRE (2008) en su artículo 78, garantiza el derecho a la no re-victimización, lo propio se plasma en las diferentes leyes y normas que conforman el ordenamiento jurídico nacional, así como en los tratados internacionales de los que el Ecuador es un estado parte y se ha ratificado.

El artículo 154 Ibídem, faculta a las Ministras y Ministros de estado la expedición de acuerdos y resoluciones administrativas que requiera su gestión. Es por ello, de allí pues, que se precisa que el DECE no intervenga con las víctimas sino hasta que Fiscalía General del Estado, disponga a la máxima autoridad de la institución educativa que a través de este departamento se brinde apoyo psicológico y emocional al estudiante agraviado, permitiendo que se actúe de oficio cuando no se ha podido identificar la infracción de tipo penal. Ahora bien en ningún momento se pretende invisibilizar el deber de denunciar que se encuentra ordenado en el artículo 472 del Código Orgánico Integral Penal (2014). 


\section{Propuesta}

Teniendo como base lo establecido en el artículo 84 de la CRE, es necesario adecuar material y formalmente los contenidos infra constitucionales a la Constitución, consiguientemente se ha verificado la necesidad de reformar el artículo No. 6 del Acuerdo Ministerial No. MINEDUC-MINEDUC-2017-00052-A, evitando de esta forma que se configure la re victimización en torno a los estudiantes agraviados, sugiriendo se lo haga en el siguiente sentido:
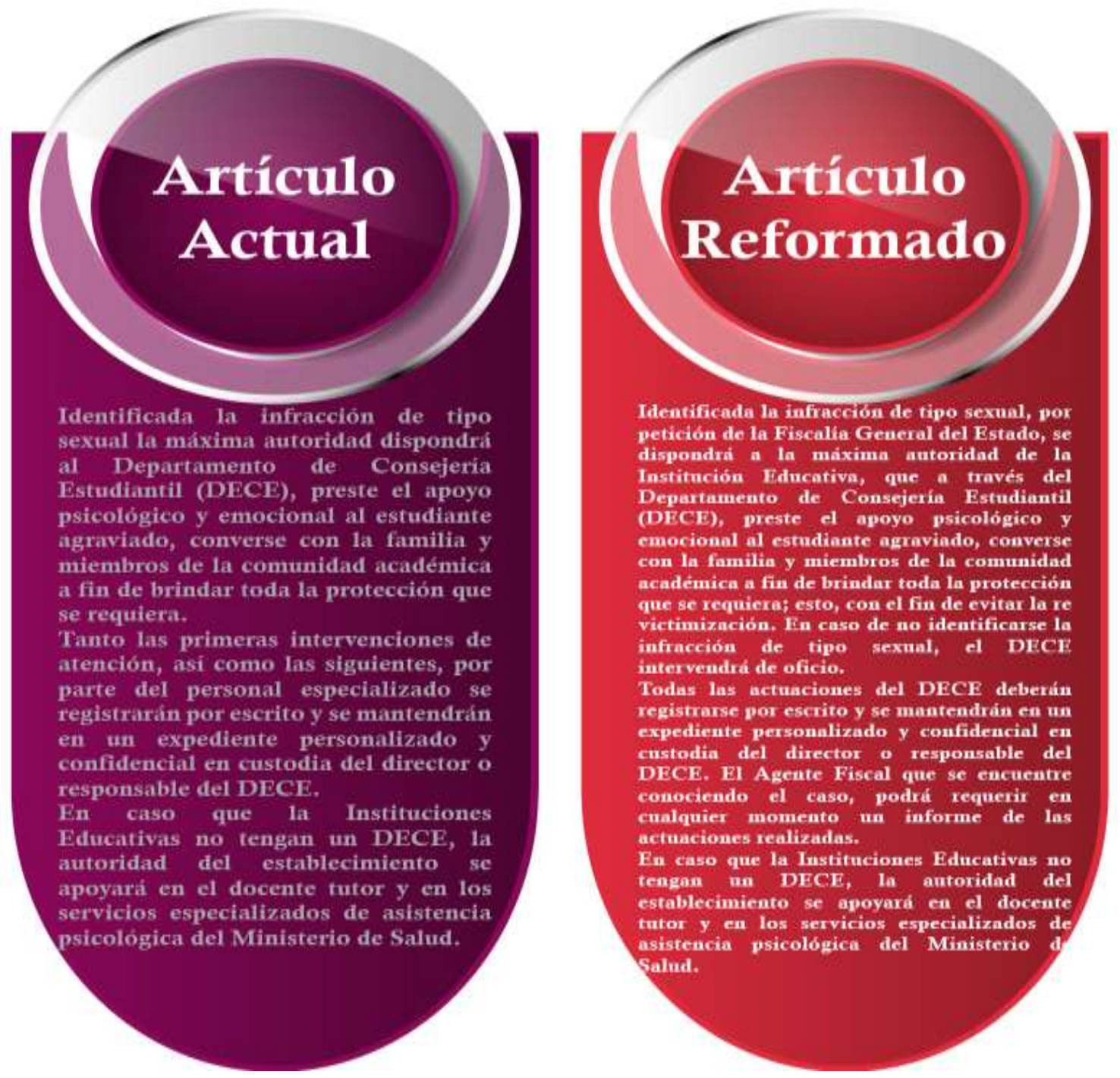

Figura 2 Propuesta de reforma al artículo No. 6 del Acuerdo Ministerial No. MINEDUCMINEDUC-2017-00052-A 


\section{DISCUSIÓN}

Los temas abordados en el presente artículo de investigación, ponen en evidencia que el artículo 6 del Acuerdo Ministerial No. MINEDUC-MINEDUC-2017-00052-A, violenta la garantía constitucional de no re-victimización, debido a que éste faculta a los funcionarios del DECE para que realicen intervenciones con los estudiantes agraviados, quienes han sufrido violencia de tipo sexual. Se observa también que existen realidades deficientes al respecto en la operación de justicia, sobre todo en la fase pre procesal de obtención de pruebas, lo cual a todas luces encasilla en la trasgresión del artículo 78 de la CRE.

En este contexto, son varias las causas que originan esta situación, constituyéndose como principal, la falta de políticas públicas claras las cuales a su vez deben ser sistemáticas por parte de las Autoridades competentes, no obstante, no se puede dejar de reconocer que han existido importantes avances en temas cuyo propósito es erradicar la violencia de género hacia las niñas, niños, adolescentes y mujeres; $\mathrm{Y}$, consecuentemente los procesos de re victimización, pero estos aun resultan insuficientes.

Cabe mencionar por otra parte, que tampoco con el presente artículo se intenta satanizar la existencia y el rol del DECE con respecto a los estudiantes agraviados por el cometimiento de un injusto penal en su contra, ya que de acuerdo a un porcentaje considerable en los resultados de las encuestas realizadas, se estima como positivo el acompañamiento y la contingencia emocional que pueden brindar a las víctimas, siempre y cuando ésta sea dispuesta por quien lleva la investigación de la causa, o sea, por un Agente Fiscal.

Atendiendo estas consideraciones, se concluye que toda intervención que se realice con respecto a las víctimas de delitos sexuales, debe ser mínimamente lesiva sea en el aspecto físico, psicológico o moral, evitando de esta forma que con el recuento de los hechos se configure la re-victimización, a través de una innecesaria vivencia de reconstrucción de un episodio repugnante y doloroso. 


\section{FINANCIAMIENTO}

No monetario.

\section{AGRADECIMIENTO}

A los funcionarios públicos, entre agentes fiscales, secretarios de fiscalías, miembros de la Junta Cantonal de Protección de Derechos, analistas jurídicos de la Coordinación No. 6 de Educación; por el apoyo prestado en el desarrollo de este proceso investigativo.

\section{REFERENCIAS CONSULTADAS}

Asamblea Constituyente. (20 de Octubre de 2008). Constitución de la República del Ecuador 2008.[Constitution of the Republic of Ecuador 2008]Recuperado de: https://n9.cl/hd0q. Garantías Constitucionales. Montecristi, Manabí, Ecuador: Registro Oficial No. 449. Recuperado el 16 de OCTUBRE de 2018, de Fiel Web (www.fielweb.com)

Asamblea Nacional. (10 de febrero de 2014). Código orgánico integral penal. [Comprehensive penal code]. Recuperado de: https://n9.cl/gn1m. Libro Preliminar. Quito, Pichincha, Ecuador: Registro oficial.

Canosa-Usera, R. (2006). El derecho a la integridad personal. [The right to personal integrity]. Recuperado de: https://n9.cl/o38rj. Valladolid: Lex Nova.

Caso V.R.P., V.P.C. y otros vs. Nicaragua. [V.R.P., V.P.C. and others vs. Nicaragua]. Recuperado de: https://n9.cl/riOs, 1 (Corte Interamericana de Derechos Humanos 08 de marzo de 2018).

Chiriboga, G., \& Salgado, H. (1995). Derechos fundamentales en la constitución Ecuatoriana. [Fundamental rights in the Ecuadorian constitution] Recuperado de:https://n9.cl/na3i. Quito: Instituto Latinoamericano de Investigaciones Sociales.

Congreso Nacional del Ecuador. (3 de Enero de 2003). Código de la Niñez y Adolescencia [Childhood and Adolescense Code]. Quito, Pichincha, Ecuador: Registro Oficial 737. Obtenido de https://www.igualdad.gob.ec/wpcontent/uploads/downloads/2017/11/codigo_ninezyadolescencia.pdf 
Dupret, M., \& Nathalia, U. (2013). Revictimización de niños y adolescentes tras denuncia de abuso sexual. [Revictimization of children and adolescents after reporting sexual abuse] Recuperado de: https://n9.cl/pllp0. Universitas, 101-128.

Erazo-Álvarez, J. C., \& Narváez-Zurita, C. I. (2020). Medición y gestión del capital intelectual en la industria del cuero - calzado en Ecuador. [Measurement and management of intellectual capital in the leather industry - footwear in Ecuador]. Revista Arbitrada Interdisciplinaria Koinonía, 437-467. doi:http://dx.doi.org/10.35381/r.k.v5i9.662

Falconí-Benítez, F. (28 de julio de 2017). Acuerdo No. MINEDUC-MINEDUC-201700052-A. Quito, Pichincha, Ecuador: Registro Oficial .

García , C., Guedes , A., \& Knerr, W. (2013). Comprender y abordar la violencia contra las mujeres. [Understand and address violence against women] Recuperado de:https://n9.cl/q4br. Washington,DC: Organización mundial de la salud.

Gómez-Luna, E., Fernando-Navas, D., Aponte-Mayor, G., \& Betancourt-Buitrago, L. (2014). Metodología para la revisión bibliográfica y la gestión de información de temas científicos, a través de su estructuración y sistematización. [Methodology for bibliographic review and information management of scientific topics, through its structuring an. Dyna, 158-163.

Hernández-Sampieri, R., Collado, C., \& Baptista, M. (2014). Metodología de la Investigación [Investigation methodology]. México D.F.: McGraw-Hill / Interamericana Editores S.A. de C.V.

López-Contreras, R. (2015). Interés superior de los niños y niñas: Definición y contenido. [Best interests of boys and girls: Definition and content]. Revista latinoamericana de ciencias sociales, niñez y juventud, 51-70. doi:10.11600/1692715x.1311210213

Marquez-Cárdenas, A. (2011). La victimilogía como estudio. Redescubrimiento de la víctima para el proceso penal.[Victimology as a study. Rediscovery of the victim for the criminal process]. Recuperado de: https://n9.cl/n22p. Polegómenos. Derechos y Valores, 27-42.

Moscoso-Parra , R., Correa-Calderón , J., \& Orellana-Izurieta , G. (2018). El derecho constitucional a la no revictimización de las mujeres en el Ecuador. [The constitutional right to non-revictimization of women in Ecuador]. Recuperado de: https://n9.cl/kue9c. Revista universidad y sociedad, 60-68. 
Mireya Paulina Cabrera-Marín; Juan Carlos Erazo-Álvarez; Cecilia Ivonne Narváez-Zurita; Camilo Emanuel Pinos-Jaén

Organización de las Naciones Unidas. (20 de noviembre de 1989). Convención de los derechos del niño. [Convention of children's rights]. Obtenido de https://www.unicef.es/sites/unicef.es/files/CDN_06.pdf

Rodríguez, A. (1 de Julio de 2017). Métodos científicos de indagación y construcción del conocimiento [Scientific methods of inquiry and knowledge construction]. Revista Escuela de Administración de Negocios, 1-26. doi:https://doi.org/10.21158/01208160.n82.2017.1647

Rodríguez, M. F. (2011). La supremacía constitucional: naturaleza y alcances. [Constitutional supremacy: nature and scope]. Recuperado de: https://n9.cl/vkug. Díkaion, 97-117.

Romero-Heras, J. C., Erazo-Álvarez, J. C., Pinos-Jaén, C. E., \& Narváez-Zurita, C. I. (2020). Desnaturalización de acciones afirmativas en el acceso a la educación superior en el Ecuador a favor de las víctimas de violencia de sexo o género [Denaturation of affirmative actions in access to higher education in Ecuador in favor of victims of sex] . Iustitia Socialis, 50-72. doi:http://dx.doi.org/10.35381/racji.v5i8.561

Torres-Pérez, S. E., Narváez-Zurita, C. I., Pinos-Jaén, C. E., \& Erazo-Álvarez, J. C. (2020). El derecho a la adopción de parejas del mismo sexo: El caso ecuatoriano [The right to adoption of same-sex couples: The Ecuadorian case]. Iustitia Socialis, 22-41. doi:http://dx.doi.org/10.35381/racji.v5i1.599

UNICEF Comité Español. (20 de noviembre de 1989). Convención sobre los derechos del niño. [Children's rights convention] Recuperado de:https://n9.cl/pnq7. Convención sobre los derechos del niño. Madrid, Madrid, España: Nuevo Siglo.

Villabella, C. (2015). Los métodos en la investigación jurídica. Algunas Presiciones [Methods in legal research. Some Presitions]. Biblioteca Jurídica Virtual del Instituto de Investigaciones Jurídicas de la UNAM, 921-953. Obtenido de https://archivos.juridicas.unam.mx/www/bjv/libros/8/3983/46.pdf

Zhindón-Idrovo, J. V., Erazo-Álvarez, J. C., Pozo-Cabrera, E. E., \& Narváez-Zurita, C. I. (2020). La desnaturalización de la acción extraordinaria de protección en la práctica judicial ecuatoriana [The denaturation of the extraordinary protection action in the Ecuadorian judicial practice]. Iustitia Socialis, 390. doi:http://dx.doi.org/10.35381/racji.v5i8.579 
Iustitia Socialis. Revista Arbitrada de Ciencias Jurídicas.

$$
\text { Año V. Vol. V. N9. Julio - Diciembre } 2020
$$

Hecho el depósito de Ley: FA2016000064

ISSN: $2542-3371$

FUNDACIÓN KOINONIA (F.K). Santa Ana de Coro, Venezuela

Mireya Paulina Cabrera-Marín; Juan Carlos Erazo-Álvarez; Cecilia Ivonne Narváez-Zurita; Camilo Emanuel Pinos-Jaén

(C2020 por los autores. Este artículo es de acceso abierto y distribuido según los términos y condiciones de la licencia Creative Commons Atribución-NoComercial-Compartirlgual 4.0 Internacional (CC BY-NC-SA 4.0) (https://creativecommons.org/licenses/by-nc-sa/4.0/). 